\title{
Patent Foramen Ovale and Cryptogenic Stroke
}

\author{
J Ahmed \\ Cardiologist, The Prince Charles Hospital, Brisbane, Australia.
}

(Cardiovasc. j. 2010; 3(1) : 81-84)

Stroke is a major cause of morbidity and mortality. The majority of ischemic strokes are due to cardioembolism, athero-embolism from large vessels or occlusive diseases of the small cerebral vessels (lacunar stroke). Many strokes occur without a well defined aetiology and are known as cryptogenic stroke (CS). This accounts for about 30 to 40 percent of ischemic strokes. ${ }^{1}$

Stroke aetiology may be classified according to the Trial of ORG 10172 in Acute Stroke Treatment (TOAST) criteria. ${ }^{2}$ As per TOAST classification cryptogenic stroke is defined as stroke not attributable to definite cardioembolic source, large artery atherosclerosis or small artery disease. Cryptogenic stroke includes patients with less wellestablished potential causes of cardiac embolism, such as: patent foramen ovale (PFO), aortic arch atheroma and mitral valve strands.

\section{Cryptogenic stroke and PFO}

Studies have demonstrated an increased incidence of PFO and Atrial septal aneurysm (ASA) in patients classified as having cryptogenic stroke. However, the role of the PFO and ASA in stroke aetiology remains controversial.

Anatomy of patent foramen ovale: The foramen ovale is a flap-like valve between the right and left atrium and is an important component of the fetal circulation. After birth, a relative increase in left atrial pressure closes the flap, and adhesions frequently form a structurally intact atrial septum. However, in approximately 25 percent of adults, the foramen ovale remains patent and acts a potential right-to-left shunt.

\section{Diagnosis of PFO}

A PFO is usually detected by transthoracic echocardiography (TTE) or Transesophageal echocardiography (TOE), especially when performed with agitated saline contrast injected during a valsalva manoeuvre. The diagnosis is established by demonstration of an interatrial communication with right to left transit of contrast microbubbles within 3 to 4 cardiac cycles of maximum right atrial opacification (Figure $1 \& 2$ ).

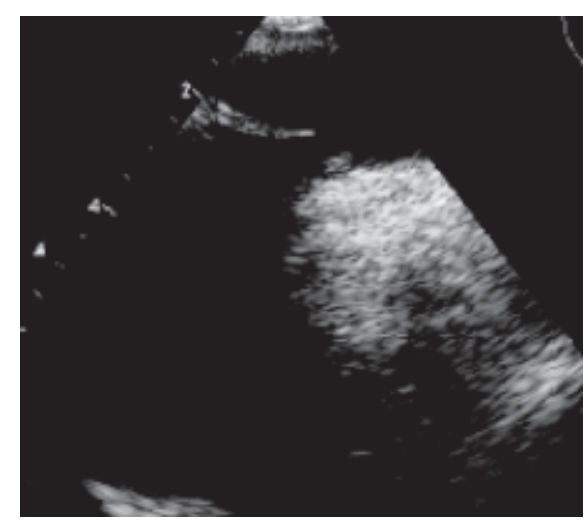

Fig.-1: Transesophageal echocardiogram systolic view showing patent foramen ovale.

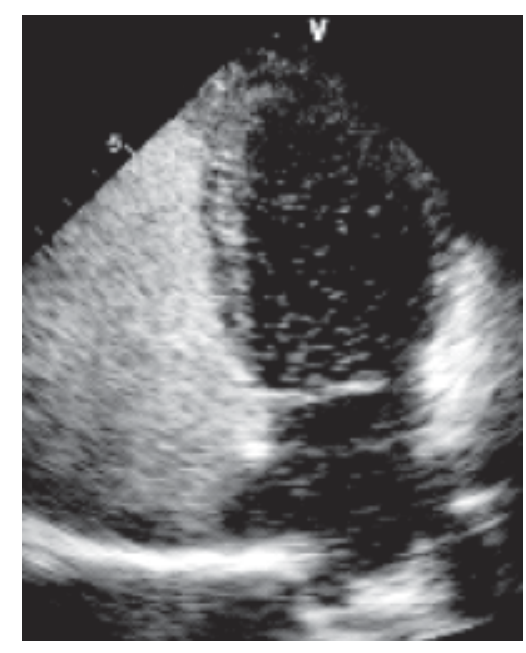

Fig.-2: 2D agitated saline contrast study showing passage of bubble from right to left consistent with PFO.

Kerr et al described a new Transmitral Doppler technique for PFO detection. It is a sensitive and specific method for TTE PFO detection and allows quantification of right to left bubble passage and 
obviates the need for TOE in many patients after stroke. ${ }^{3}$ In this method the transthoracic bubble echocardiographic study is repeated with transmitral pulse wave Doppler recorded at the mitral valve tip, the gain settings are reduced so that normal trace is only just visible, individual bubble signals entering the left heart chambers result in bright signal in Doppler trace. The size of the PFO may be estimated using a validated visual bubble score. Images are taken at rest and after valsalva manoeuvre (Figure 3 ).

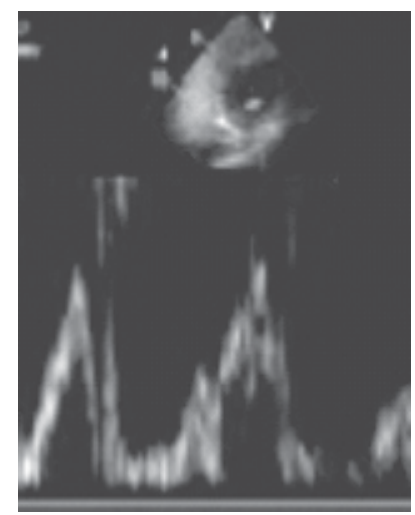

Fig.-3: Transmitral doppler showing bright signals representing passage of bubble across mitral valve.

\section{Atrial septal aneurysm}

An atrial septal aneurysm is defined as a redundant and hypermobile portion of the interatrial septum that demonstrates more than $10 \mathrm{~mm}$ excursion from the centreline during the cardiac cycle.

\section{Prevalence of Patent Foramen Ovale}

In an autopsy series of 965 patients PFO was identified in $27 \%$ of patients with normal heart. In that autopsy series its prevalence appeared to decline with age. ${ }^{4}$ In a study using contrast echocardiography PFO was detected in $14.9 \%$ of 1100 stroke free subjects older than 39yrs of age.

An atrial septal aneurysm was present in $2.5 \%$ of total cohorts, most often in association with PFO. ${ }^{5}$ Meissner et al using transesophageal echocardiography reported a $24.3 \%$ prevalence of PFO among 585 randomly sampled patients, 45 years of age or older, participating in the Stroke Prevention Assessment of Risk in a community (SPARC) study. An atrial septal aneurysm was present in $1.9 \%$ of the subjects, including $4.3 \%$ of those with PFO's. ${ }^{6}$
Potential mechanisms of cryptogenic stroke in PFO and ASA include paradoxical embolism from a venous source, direct embolisation of thrombus formed within the PFO or an associated ASA, passage of vasoactive humoral substances that escape pulmonary degradation, and thrombus formation caused by atrial arrhythmias, such as paroxysmal atrial fibrillation.

\section{Association between CS and PFO}

Most but not all observational studies reported a higher prevalence of $\mathrm{PFO}$ among patients with CS than among normal control subjects and among patients in whom a cause of stroke could be identified. The association between PFO and CS has been more convincingly demonstrated in younger (less than 55 years of age) versus older patients ( 55 years of age or older). Lamy et at detected a PFO with transesophageal echocardiography in $45.9 \%$ of 581 patients 55 years of age or younger. ${ }^{7}$ In PICCS study PFO was present in $33.8 \%$ of patients 30 to 85 years of age. ${ }^{8}$ Handke et al reported a statistical association between $\mathrm{PFO}$ and $\mathrm{CS}$ in both younger and older patients. $\mathrm{PFO}$ was present in $43.9 \%$ in younger CS patients versus $14.3 \%$ in younger patients with known cause. In patients older than 55 years of age PFO was present in $28.3 \%$ compared with $11.9 \%$ with a stroke of known cause. ${ }^{9}$

Some studies failed to demonstrate a strong association between PFO and stroke. In Northern Manhattan study (NOMAS), PFO was not associated with increased risk of stroke in a multiethnic cohort of both men and women or in patients younger or older than 60yrs. ${ }^{5}$ In SPARK study PFO was not an independent risk among normal subjects older than $45 \mathrm{yrs} .{ }^{6}$

\section{Larger the size of PFO, greater the risk?}

Many studies have implicated an increased risk of stroke with anatomic size of $\mathrm{PFO}$ or magnitude of the shunt and the coexistence of ASA, but these associations has not been observed consistently.

\section{Treatment}

The best treatment modality to prevent recurrent stroke in patients with PFO has not been defined. 
Treatment modalities include medical therapy, with antiplatelet or anticoagulant agents, percutaneous device closure or open surgical repair. Whereas suture closure of an incidental PFO is performed routinely during the course of an operation undertaken for another indication, primary surgical repair is rarely advocated. The choice between medical therapy and percutaneous device closure has been a subject of intense debate over last few years. ${ }^{10} \mathrm{Few}$ nonrandomized clinical trials suggested lower rates of recurrent stroke after device closure of PFO, especially among patients with coexistent atrial septal aneurysm. There are not enough randomised clinical trials comparing the relative safety and efficacy of the two methods and the issue remains unresolved. There are few ongoing clinical trials comparing medical therapy and percutaneous device closure, but enrolment in these studies has been lagging causing delay in completion.

Medical therapy for secondary prevention in PFO patients with cryptogenic stroke includes, antiplatelet agents such as aspirin or anticoagulant agent such as warfarin. Both agents may have similar efficacy. In PICSS study, patients were treated with aspirin or warfarin. The 2 year primary event rate for all cause death or recurrent ischemic stroke with warfarin was not significantly different from those treated with aspirin. ${ }^{11}$ Whereas Cojec et al reported that warfarin may be more effective than anti-platelet therapy in secondary stroke prevention. ${ }^{12}$.

The percutaneous closure of PFO is a minimally invasive non-surgical procedure. Usually femoral vein cannulation is done for device delivery. Flouroscopy and TOE is commonly used to guide device implantation. Complications include death, cardiac tamponade, haemorrhage, need for surgical intervention, pulmonary embolism, periproceduaral atrial arrhythmia, transient AV block, device arm fracture, device embolization, AV fistula formation and femoral haematoma. There are few devices available for percutaneous closure of PFO. Commonly used devices are Amplatzer PFO occluder device (figure 4), Starflex septal closure system and GORE HELEX septal occluder device.

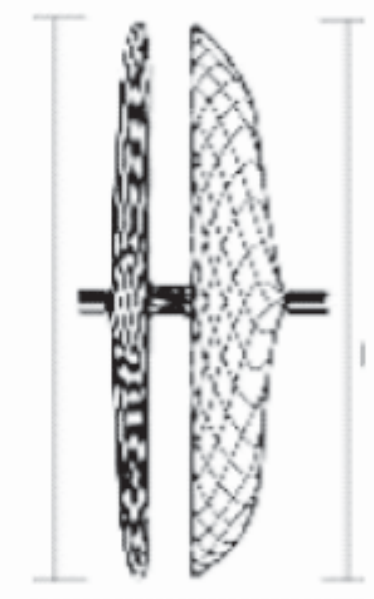

Fig.-4: Amplatzer PFO closure device.

\section{Conclusion:}

PFO is a common occurrence, occurring in about $25 \%$ of population. Studies have demonstrated an increased prevalence of PFO in cryptogenic stroke patients. There is no consensus on optimum strategy of secondary prevention. Systematic review of nonrandomized studies suggested that a substantial proportion of recurrent thrmboembolic event might be prevented by implantation of PFO closure device compared with medical therapy. ${ }^{13}$ Moreover, transcatheter closure of $\mathrm{PFO}$ appeared to be safe with a major complication rate of less than $2 \%$. But there are not enough randomised controlled trials to compare medical therapy and PFO device closure. Present guidelines recommend medical therapy with antiplatelet agents, unless there is another indication for warfarin therapy, after first stroke. But device closure may be considered for patients with recurrent stroke despite optimal medical therapy. ${ }^{14}$

\section{References:}

1. Sacco RL; Ellenberg JH; Mohr JP; Tatemichi TK; Hier DB; Price TR; Wolf PA. Infarcts of undetermined cause: the NINCDS Stroke Data Bank. Ann Neurol 1989 Apr;25(4):382-90.

2. Adams HP Jr, Bendixen B, Kappelle LJ, et al. Classification of subtype of acute ischemic stroke: definitions for use in a multicenter clinical trial. Stroke 1993;24:35- 41.

3. Kerr AJ; Buck T; Chia K; Chow CM; Fox E; Levine RA; Picard MH. Transmitral Doppler: a newtransthoracic contrast method for patent foramen ovale detection and quantification. J Am Coll Cardiol 2000 Nov 15;36(6):1959-66. 
4. Hagen PT, Scholz DG, Edwards WD. Incidence and size of patent foramen ovale during the first 10 decades of life: an autopsy study of 965 normal hearts. Mayo Clin Proc 1984; 59: 17-20.

5. Di Tullio MR, Sacco RL, Sciacca RR, et al. Patent foramen ovale and the risk of ischemic stroke in a multiethnic population. J Am Coll Cardio 2007; 49: 797-802.

6. Meissner I, Khandheria BK, Heit JA, et al. Patent foramen ovale: innocent or guilty? Evidence from a prospective population-based study. J Am Coll Cardiol 2006; 47: 440-5.

7. Lamy C, Giannesini C, Zuber M, et al. Clinical and imaging findings in cryptogenic stroke patients with and without patent foramen ovale: the PFOASA Study. Stroke 2002; 33: 706-11.

8. Homma S, Sacco RL, Di Tullio MR, et al. Effect of medical treatment in stroke patients with patent foramen ovale: Patent Foramen Ovale In Cryptogenic Stroke Study. Circulation 2002; 105: 2625-31.

9. Handke M, Harloff A, Olschewski M, et al. Patent foramen ovale and cryptogenic stroke in older patients. N Engl J Med 2007; 357: 2262-8.
10. O'Gara P, Messe SR, Tuzcu EM, et al. Percutaneous Device Closure of Patent Foramen Ovale for Secondary Stroke Prevention. A Call for Completion of Randomized Clinical Trials. Circulatio 2009;119:27432747.

11. Homma S, Sacco RL, Di Tullio MR, et al. Effect of medical treatment in stroke patients with patent foramen ovale: Patent Foramen Ovale In Cryptogenic Stroke Study. Circulation 2002; 105: 2625-31.

12. Cujec B, Mainra R, Johnson DH, et al. Prevention of recurrent cerebral ischemic events in patients with patent foramen ovale and cryptogenic strokes or transient ischemic attacks. Can J Cardiol 1999; 15: 5764 .

13. Khairy P, O'Donnell CP, Landzberg MJ. Transcatheter closure versus medical therapy of patent foramen ovale and presumed paradoxical thromboemboli: a systematic review. Ann Intern Med 2003; 139: 753-60.

14. Sacco RL, Adams R, Albers G, et al. AHA/ASA Guidelines. Guidelines for Prevention of Stroke in Patients With Ischemic Stroke or Transient Ischemic Attack. Stroke 2006;37:577. 